\title{
Can precision electrophile signaling make a meaningful and lasting impression in drug design?
}

\author{
Marcus J. C. Long ${ }^{\ddagger}$, Amogh Kulkarni†, and Yimon Aye+* \\ †Swiss Federal Institute of Technology in Lausanne (EPFL), 1015 Lausanne, Switzerland \\ ‡University of Lausanne, 1015 Lausanne, Switzerland \\ *Correspondence: yimon.aye@epfl.ch
}

\begin{abstract}
Summary
For several years, drugs bearing reactive electrophilic appendages have been developed. These units typically confer prolonged residence time of the drugs on their protein targets, and may assist targeting shallow binding sites and/or improving drug-protein target spectrum. Studies on natural electrophilic molecules have indicated that in many instances natural electrophiles use similar mechanisms to alter signaling pathways. However, natural reactive species are also endowed with other important mechanisms to hone signaling properties that are uncommon in drug design. These include ability to be active at low occupancy and elevated inhibitor kinetics. Here we discuss how we have begun to harness these properties in inhibitor design.
\end{abstract}

Keywords: electrophile signaling; cysteine; LDE; HNE; T-REX

\section{Perks of covalent binders?}

Many drugs interact with their target reversibly. This class includes several seminal drugs, like Gleevec ${ }^{[1]}$ and Gefitinib, ${ }^{[2]}$ as well as recent drugs, including midostaurin, ${ }^{[3]}$ and ozanimod, ${ }^{[4]}$ that treats relapsing multiple sclerosis (Figure 1). The continued development of non-covalent drugs testifies to their ongoing importance, and further that small molecules remain the bread and butter of drug design. Nevertheless, reversible binders in clinical use have several core similarities, giving way to common issues. They obligatorily bind targets with high affinity, and hence typically need a relatively large interaction surface with target proteins, which can favor molecules with poor drug properties. Their binding is significantly affected by the presence of competing ligands, which act to lower the amount of inhibited complex present at equilibrium. Furthermore, as many of the chosen targeted binding sites, e.g., ATP-binding site within kinases, share similarity, these drugs can have off-target effects that require continuous monitoring (e.g., Dasatinib ${ }^{[5]}$ ). Although high-affinity non-covalent drugs often do not release immediately from their targets post removal of the drug from the media or clearance from a patient, upon lowering of effective drug concentration, these drugs ultimately dissociate from their target protein, which then continues to contribute to the disease state. Essentially, these drugs need relatively ideal conditions to make an impression on their target, and, if those conditions are not sustained, the impression made may be fleeting. 
Covalent drugs, conversely, require only a brief encounter with their target to imprint on their target. Thus, be the binding site partly occupied by a competing ligand, or be it "shallow", covalent drugs can still get to work, time-dependently forming a permanent bound state. Indeed, drugs like Tecfidera (dimethyl fumarate), ${ }^{\left[{ }^{[6]}\right.}$ and drugs in clinical trials like phenethyl-isothiocyanate ${ }^{[7]}$ do not ostensibly form an encounter complex with their targets on the way to forming a covalent bond (Figure 1). Regardless of the mechanism of labeling, once formed, the covalent bond typically ensures that there is no turning back; new protein synthesis is typically needed to reinitialize activity. However, making this sort of impression requires the stars to align: next to the site of labeling/binding site, a reactive residue must be present to form the covalent bond; otherwise, the targeted residue must be particularly nucleophilic. These stringent requirements explain another important win, particularly for ligandable covalent binders: their proclivity to label similar proteins, even those with highly similar binding sites, is not equal. Although there are numerous examples, e.g., $\operatorname{ras}^{G 12 C}$, an oncogenic driver mutant of ras, stands out. Only this mutant, and not the wild-type allele, or similarly oncogenic alleles, e.g., $\operatorname{ras}^{G 12 \mathrm{~V} / \mathrm{D}}$, is targeted by the electrophilic inhibitors, MRTX849 ${ }^{[8]}$ and AMG510 ${ }^{[9]}$, now in clinical trials. $^{[10],[11]}$ In this way, MRTX849 and AMG510 are high precision therapies to target cancer cells expressing the ras $^{G 12 C}$ mutation. Indeed, druggable cysteines are now at a premium, ${ }^{[12],[13]}$ and once identified, a druggable cysteine residue can give way to numerous approved drugs. 


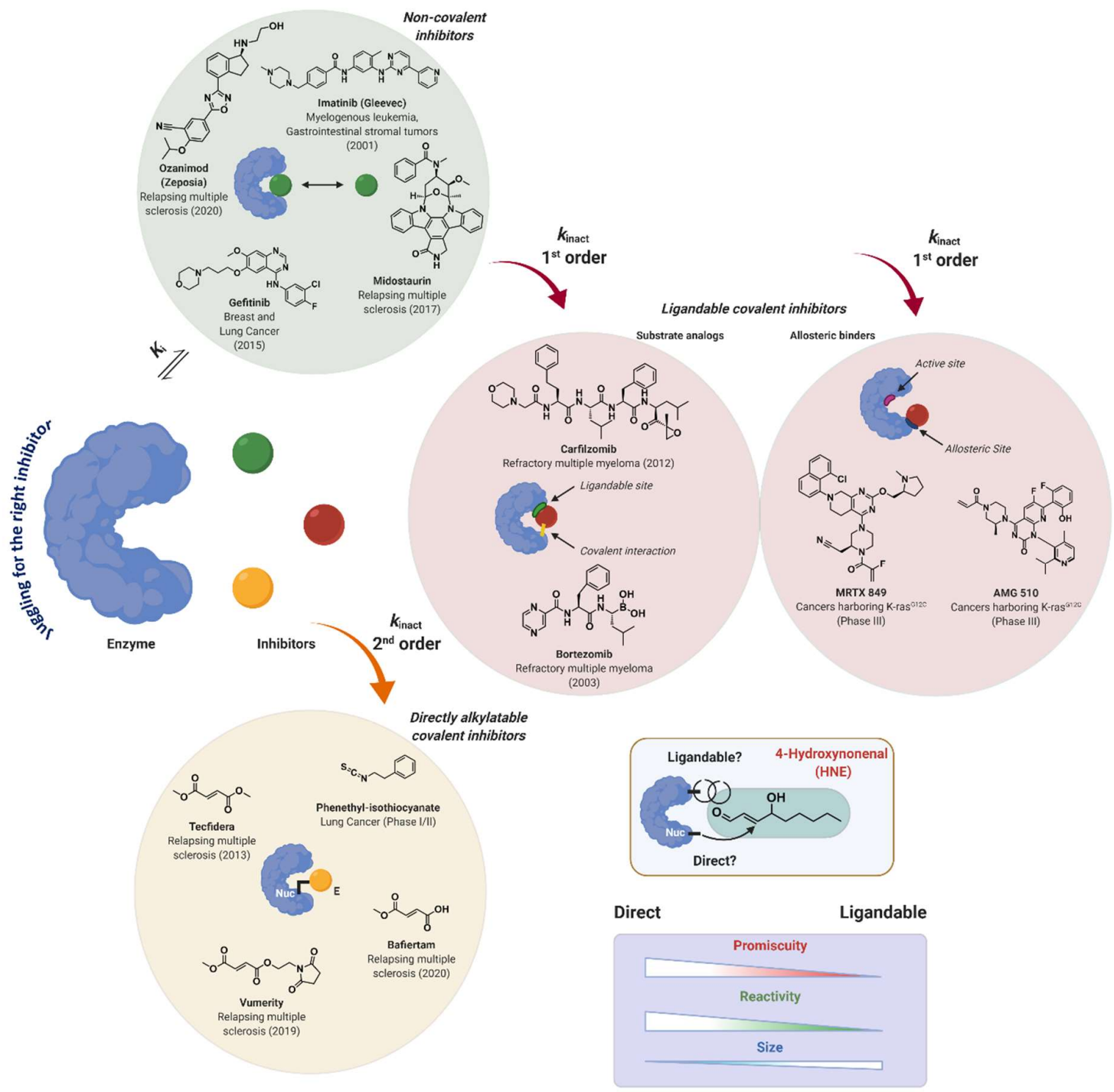

Figure 1. Juggling for the right inhibitor. Inhibitor classification exemplified by emerging therapeutics in clinical use or trials. (Inset, top: HNE: Can there be a ligandable approach for identifying HNE-sensors?; bottom: Relationship between promiscuity, reactivity, size of the Inhibitor, as a function of its mode of interaction with target, either direct adduction or ligandable interaction. It is unclear which mechanism HNE uses to interact with each specific target, although the best HNE sensors likely show some degree of ligandable contribution to HNE labeling. ${ }^{[14]}$ 


\section{Could we do better?}

Obviously from whatever perspective we approach covalent inhibition, there are manifold potential benefits compared to traditional, reversible binders. It is noteworthy that not all covalent binders will show all the perks above. There are instances where the impact of covalent binding can be dumbed down, and the inherent reactivity of covalent binders can lead to significant off-target effects. Nevertheless, given that cysteine targeting is such big business, we were struck that targeting a specific cysteine is often a matter of happenstance: either in a series of potential targets, one has a cysteine in the correct position to allow a covalent interaction, or a mutant is formed that happens to introduce a cysteine poised to form a covalent bond. Could we do better?

For inspiration, we could consider substrate analogs. Indeed, this is one of the most venerable classes of drugs, e.g., penicillin, a covalent inhibitor of DD-transpeptidase, an enzyme essential for bacterial cell-wall synthesis. ${ }^{[15]}$ At least three anti-cancer drugs exist in this category, including, bortezomib ${ }^{[16]}$ and carfilzomib, ${ }^{[17]}$ approved covalent proteasome inhibitors. Such reactive inhibitors target intrinsically-reactive residues chemically essential for enzyme catalysis. Most of these drugs target hard nucleophilic residues for covalent ligandability; enzymes bearing softer nucleophiles, e.g., cysteine proteases and other nucleophilic active-site cysteines, have proven harder to drug. Nevertheless, the unambiguity of modifying the catalytically-essential residue of the enzyme and the benefits in terms of possibilities for substrate-analog design and kinetic favorability that can be so derived, render these types of inhibitors appealing. Thus, these examples raise an interesting question: rather than targeting cysteines of convenience, are there cysteines in the proteome that are ostensibly not involved in catalysis, but manifest privileged responsivity to small-molecule electrophiles that may be applicable to drug design?

Data concerning such cysteines have been around in several guises for several years. Seminal work from Herman Esterbauer ${ }^{[18-22]}$ and later Earl Stadtman ${ }^{[23-26]}$ showed that lipid-derived electrophiles (LDEs) could react with specific proteins and usher changes in reactivity of specific enzymes upon labeling by LDEs. Slowly it came into focus that LDEs were reactive with off-active-site cysteines in many instances. It was really not until the pioneering work of Cravatt ${ }^{[27-31]}$ that more started to be known about how representative this reactivity was across the cysteome. Using an ingenious method albeit with numerous inherent limitations, which have been discussed elsewhere, ${ }^{[32]}$ quite astounding differences in rates in reactivity and selectivity were disclosed across a pool of $\sim 1000$ protein cysteines for seemingly-similar electrophiles. These results indicate that several cysteines can behave like active-site cysteines in terms of their reactivity and selectivity with respect to the "global" cysteine pool. Over the intervening years, new methods have been released to profile large numbers of cysteines. These have remedied some of the issues with the original method, ${ }^{[33]}$ although many have brought in other limitations including, conversion of the electrophile to a more- or differentiallyreactive specie, prior to labeling and limited chemoselectivity. ${ }^{[34]}$

But all the above electrophile-sensor-detection techniques ultimately flounder on a common stumbling block: it is difficult to demonstrate that specific protein engagement by a pleiotropic electrophile is directly responsible for phenotypes observed upon electrophile treatment. The current gold standard is that the target cysteine be mutated to a non-nucleophilic residue and suppression of the specific phenotype be examined in this mutant background. This experiment is usually eschewed in favor of simpler-to-perform in vitro experiments, and when it is performed, it often fails. However, as electrophile-dosing regimens upregulate numerous knock-on stress signals [including reactive oxygen species (ROS) and other reactive electrophilic species (RES), e.g., LDEs], ${ }^{[35]}$ as many profiling 
methods assign labeling only indirectly, and mass-spectrometry techniques coupled to these methods do now allow determination of ligand occupancy, what is causing the phenotypes-e.g., electrophile labeling, ROS-adduction, pathway inhibition/stimulation-is not clearly discernible.

\section{The benefits of being 'on target'}

Mindful of these inherent limitations, we established a program investigating precision proteinelectrophile modification. This method, T-REX, ${ }^{[36]}$ has proven that single protein-electrophile engagement can trigger electrophile signaling. T-REX leverages a novel mechanism to usher a native or synthetic electrophile of choice to a specific protein of interest (POI) giving that prechosen target $\mathrm{POI}$ the opportunity to react with the specified electrophile prior to other proteins within the cell (or organism). Such a mechanism is achieved by photocaging the electrophile and fusing the chosen POI to a chemically-modifiable protein tag, such as Halo, to which the photocaged electrophile can become anchored (Figure 2) ${ }^{[37]}$ Although T-REX cannot prove that a specific electrophile labeling event occurs in living systems, T-REX allows interrogation of the ramifications of precision-labeling events and gives precise controls to account for off-target effects (unavailable in other methods). T-REX has shown that even in unstressed cells-with no ostensible depletion of glutathione nor electrophile labeling on the rest of the proteome-protein-specific electrophile engagement can re-route signaling pathways. This finding validated the many years of work examining protein labeling by 4hydroxynonenal (HNE, a quintessential LDE) in vitro/in HNE-bolus-dosed cells. However, we found that proteins previously implicated in electrophile sensing were not always particularly sensitive to T-REX or in vitro dosing with low concentrations of LDEs. ${ }^{[3],[38]}$ Conversely, novel LDE-sensor proteins identified using T-REX, emerged to be much more reactive to LDEs in vitro than the previously identified proteins. Although an exhaustive comparison is yet to be enacted, we infer that REX technologies collectively identify particularly LDE-sensitive proteins. One such sensor is Akt3. Akt3 is interesting because it is labeled more efficiently by HNE than the other Akt isoforms, Akt1 and Akt2 (the trine share $>70 \%$ identity). Performing T-REX on Akt3 led to selective suppression of Akt3activity. ${ }^{[39]}$ This suppression of activity occurred through specific labeling of C119, a residue that is unique to Akt3, and lies away from the kinase active site. However, this was not observed for Akt2 or Akt3(C119S) wherein the cysteine that senses HNE is mutated to serine (C119 is unique to Akt3, accounting for Akt3's unique labeling by HNE). Thus, profiling electrophile signaling is not only an access point to reactive cysteines, but also to isoform-selective activity modulation. This ability to mimic electrophile-protein engagement using T-REX is emerging to be a new arrow in the quiver of drug discovery, as we outline below. 

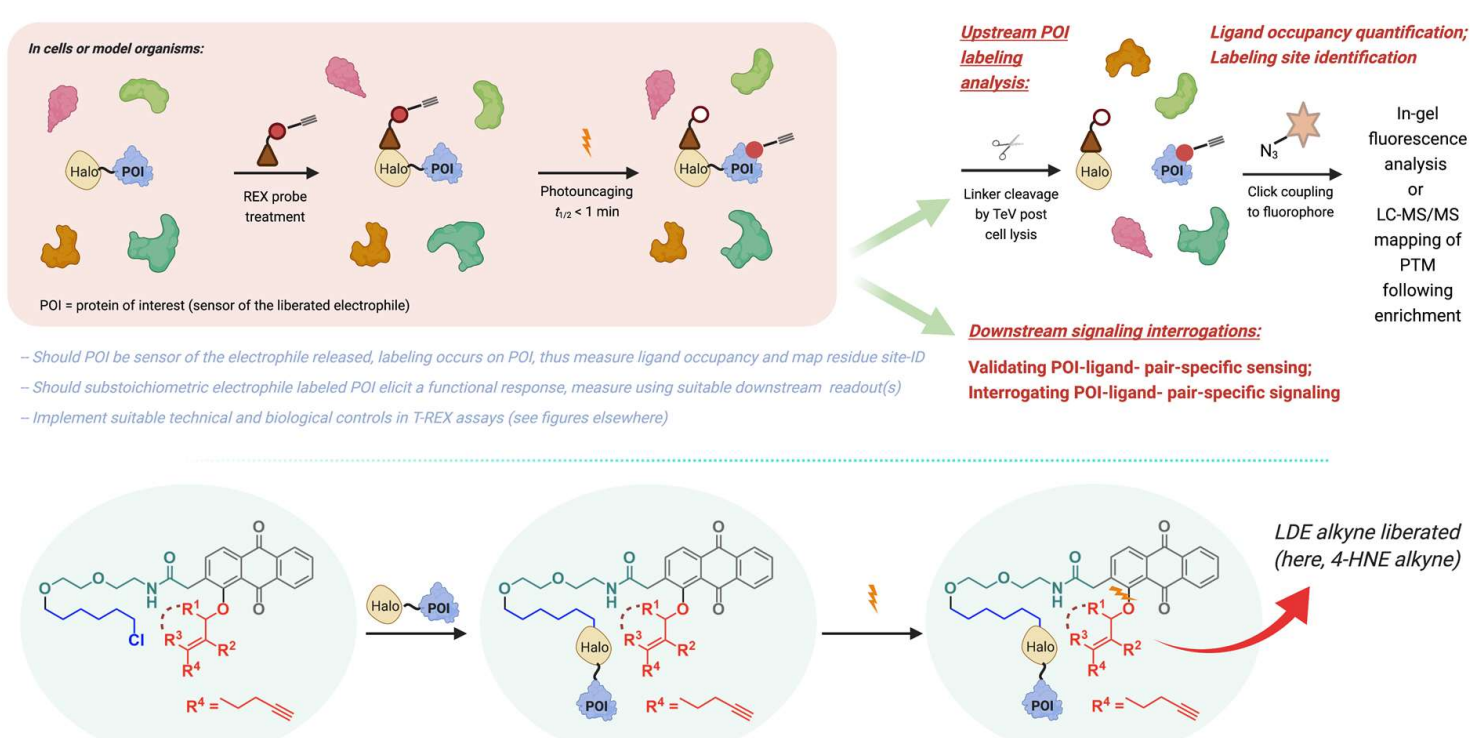

T-REX unique controls

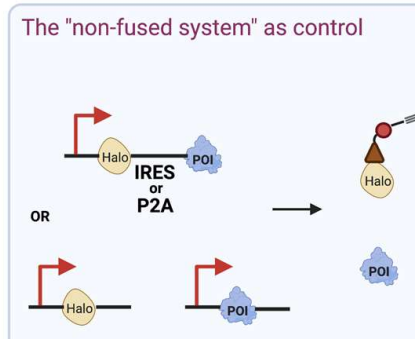

POI: RES-sensor POI (wt)

The "hypomorphic mutant" as control

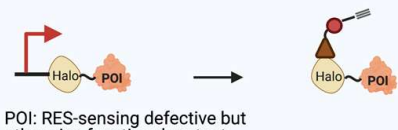

POI: RES-sensing defective but
otherwise functional mutant

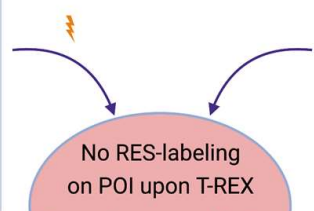

No pathway modulation upon T-REX

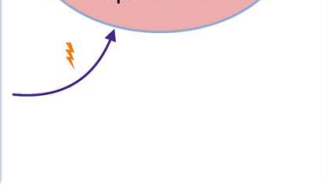

T-REX technical controls
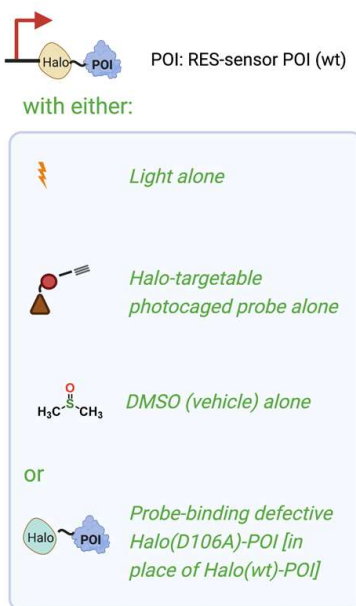

Figure 2. T-REX and associated controls. Top: workflow of T-REX highlighting the analysis of upstream target protein labeling specificity, including quantification of ligand occupancy and mapping of the residue site for the corresponding T-REX-assisted post-translational modification (PTM); as well as of downstream signaling output. Chemical scheme depicts the generic probe used in T-REX, its stoichiometric binding to Halo, and rapid $\left(t_{1 / 2}<1 \mathrm{~min}\right)$ liberation of designated alkyne-functionalized lipidderived electrophile (LDE) upon illumination. Bottom: established suite of controls that accompany T-REX assay in live cells and animals. In the 'non-fused system', encoding IRES or P2A sequence within the linker sequence in the expression plasmid or the use of two separate plasmids respectively encoding Halo and POI, allow the two units to be expressed separately in vivo/cells. Bottom left: Replicating T-REX (i.e., probe treatment and light) in this control system, rules out all potential hidden variables that could lead to artifactual signaling changes. (RES, reactive electrophilic species; IRES, internal ribosome entry site; P2A, self-cleaving peptide). If the site of modification is known, sensing-defective mutant can be constructed provided it is otherwise functional in all other aspects except electrophile sensing. Replicating T-REX on the mutant POI in place of wild-type (wt) rules out potential off-target outputs. Bottom right: technical controls of T-REX comprises omitting one of the input parameters in T-REX: i.e., omitting probe, omitting light, use of DMSO in place of DMSO solution of probe, and also the use of enzymatically-dead Halo that cannot bind probe.

\section{Target occupancy assignment, enabled by T-REX, unmasks phenotypically-dominant mode-of-action}

T-REX also allows quantitative assessment of protein ligand occupancy. ${ }^{[36]}$ Although there are many theories about how this parameter correlates with inhibition, for simple inhibitory mechanisms, ligand occupancy and inhibition should be similar. However, for Akt3 HNEylation, <20\% occupancy gave 
$>30 \%$ inhibition of total Akt activity. ${ }^{[39]}$ This hinted that HNE-mediated Akt3-inhibition was super stoichiometric, or dominant negative. Such a system can amplify inhibitor potency, and also ensure persistence of drug-induced phenotypes post inhibitor clearance and new protein synthesis; properties of an "ideal" drug. Such a mechanism could also suppress a common means by which cancer cells gain resistance to covalent drugs, cysteine mutation. This is because the heterozygotic (wt/unreactive) state retains sensitivity in a dominant-negative system, unlike a system undergoing wt-allele-specific stoichiometric inhibition. Furthermore, phylogenetic analysis implied that C119 is highly conserved, ${ }^{[13]}$ so there may be selective pressure to retain this function. We should state that how HNE exhibits dominant negative regulation of Akt3 kinase activity, or indeed many other of HNE's targets discovered by T-REX or other methods is unknown. This is an ongoing area of research for our group.

We thus pursued the hypothesis ${ }^{[13]}$ that targeting cysteines, like Akt3(C119), would present numerous added benefits in terms of kinetics, dominant-negative effects, and changes in inhibition mechanism. The obvious design for such an inhibitor was hybridizing an Akt non-covalent binder to a tamed HNElike appendage. Should the binder be an inhibitor of Akt3, such a system could behave like traditional covalent drugs, bolting the inhibitor to the protein effectively, or it could display some of the valueadded effects of HNEylation. At the limit that HNE dominates over the non-covalent binding moiety, such a system would be the antithesis of traditional inhibitor design: the non-covalent moiety of the chimeric drug would exist to deliver HNE to the protein of interest.

Our design incorporated a pan-Akt allosteric inhibitor, MK-2206, ${ }^{[40]}$ in Phase-II anticancer trials, showing 8-fold selectivity for Akt1 over Akt2, and a further 8-fold preference for Akt2 over Akt3. This scaffold is not an auspicious starting point, but we believed by disfavoring our chances for success, we could hopefully make our point more resonantly (Figure 3). However, MK-2206 is arguably the bestcharacterized allosteric Akt inhibitor. ${ }^{[41]}$ Targeting this allosteric site was necessary as the ATP-binding site-to which many Akt inhibitors bind-is too distant from C119 to promote covalent-bond formation. We fused 4-hydroxynon-2-enoic acid to MK-2206, to create our desired inhibitor, MKHNE. ${ }^{[42]}$ MK-HNE covalently labeled Akt3 preferentially over Akt2, and Akt1 was untouched. A more reactive analog, MK-FNE, showed similar preference for Akt3, but was more potent. MK-FNE and MKHNE both showed fair second order kinetics of binding, with MK-FNE approaching $k_{\text {inact }} / K_{\mathrm{i}}$ of $10^{4} \mathrm{M}^{-1} \mathrm{~s}^{-}$ 1. Interestingly, both these inhibitors were more effective, and showed significantly higher Akt3selectivity than the regioisomer MK-G, which preferentially targeted Akt2. Such divergent isoform selectivity is particularly interesting, and further investigations are currently underway. 


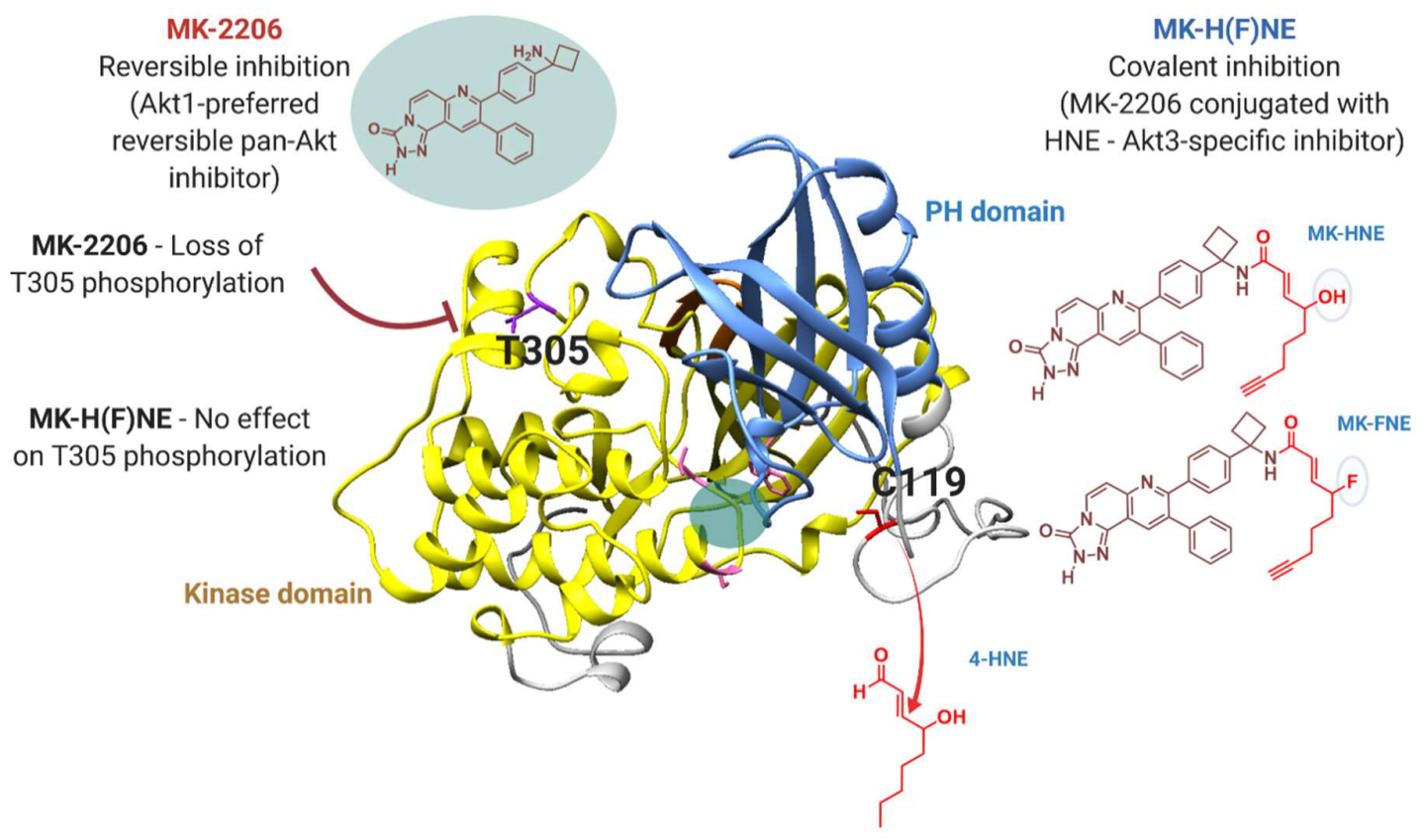

Figure 3. Development of a ligandable HNE-mimic targeting Akt3. Akt3 homology model based on Akt1 crystal structure (PDB 3096) featuring relevant inhibitors; MK-2206-binding allosteric site, common for all Akt-isoforms (blue-green circle with key residues in pink); ${ }^{[43]}$ and 4-HNE-sensing Cysteine (C119 in red).

\section{Privileged electrophile sensing extends to isoform-specific therapeutic potency}

In cell-based assays, using a FRET-based reporter for Akt activity, MK-FNE showed dominant negative, irreversible inhibition of Akt3; a 1:1 mixture of Akt3 and Akt3(C119S) showed 100\% inhibition of Akt3 activity after treatment and withdrawal of MK-FNE. Replicating the same experiment with Akt3(C119S) mixed with Akt2, showed inhibition when MK-FNE was in excess, but the effect was not dominant negative. Thus MK-HNE shows mechanistic and kinetic effects that favor Akt3 inhibition, similar to HNE.

In cells, MK-FNE and MK-HNE were potent against triple negative breast cancer (TNBC) lines and lines dependent on Akt3, but showed lower efficacy against other lines: MK-2206 was effective against most cell lines studied. In MK-FNE-sensitive cells, MK-FNE showed synergistic toxicity with Akt3 knockdown, whereas Akt1-knockdown showed synergy with Akt1. Such data are admittedly hard to interpret. However, given the known selectivity of MK-2206 for Akt1 (lines resistant to this drug upregulate Akt3 preferentially), these data are strongly consistent with MK-FNE targeting Akt3 selectively. In mouse xenograft models, using the TNBC line, MDA-MB231, MK-FNE emerged to be at least as effective as MK-2206, although MK-2206 was considerably more metabolically unstable. Critically, MK-FNE showed less blood glucose misregulation than MK-2206, a side effect known to be particularly limiting in ongoing trials involving MK-2206. These data altogether hint at a dependence of triple-negative breast cancer cells on Akt3 that merits further attention.

There were more surprises in store. MK-2206 completely suppresses Akt3 T305 phosphorylation. Conversely, HNEylation of Akt3, via T-REX, does not change T305 phosphorylation. Thus, the effects of HNE and MK2206 on Akt3, divergently affect T305 phosphorylation. We do not know whether or not this observation is related to dominant-negative effects of HNE, although we note that ATP- 
competitive Akt-inhibitors elevate phosphorylation at this residue. ${ }^{[44]}$ When we observed how MKXNE affects Akt3(T305) phosphorylation, even at saturation (a state not possible under T-REX), there was no effect on T305-phosphorylation levels. This result together with the fact that MK-HNE shows dominant-negative Akt3 inhibition, indicates that in MK-HNE the dominant effect in the bound state is the interaction between HNE and Akt3, not the interaction of MK-2206 and the protein.

\section{What is next?}

Obviously, we do not know how general such an outcome is. However, this albeit single result indicates that T-REX could be insightful in triaging drug targets, and informing on the nature of the electrophilic handle to choose. For instance, it is clear that based on phenotypes observed in T-REX-treated cells/animals, electrophile/protein targets with ideal properties (kinetics, dominant-negative effects, isoform/protein-specific targeting) can be selected and moved forward for hybrid inhibitor design. ${ }^{[45]}$ However, extrapolating somewhat, T-REX could also be useful to define phenotypic responses and the mechanism-of-action of electrophile-reversible drug hybrids, in cells or in common model organisms, in relation to, for instance, ideal off-target controls. Indeed, modeling a unique protein-drug interaction, in terms of target-intrinsic, e.g., changes in target phosphorylation, and downstream signaling consequences, in cells/organisms, is profoundly complex. Knockdown or knockout can be informative, at least for signaling consequences. However, ablation of the target protein cannot precisely inform on the electrophile-modification-induced inhibited state, even if the variation in time scales and off-target effects (which are likely as significant for knockdown/knockout as they are for compounds and as difficult to control for) between the methods are assumed to be negligible, and compound-induced gain-of-function behavior are ignored. Examining responses to the drug in knockdown or knockout lines is another possibility. However, this is once again heavily reliant on how the knockdown/knockout affects cells, and ignores target-intrinsic effects. Mutagenesis or resistantline generation can certainly be informative of a target, but in reality, these are not always performed, and can lead to complex outputs. But with T-REX we can define effects of drug-target engagement, in an otherwise-unperturbed environment, defined by specific controls. This allows us to predict drug mechanism, hone-in on desired phenotypes, all in a system that is relatively simple to manipulate.

Going a stage further, one can readily imagine comparing data from T-REX to phenotypes observed upon treatment with a hybrid drug and using comparisons to investigate/propose off-target effects of the hybrid drug. Such experiments can synergize well with traditional proteomics experiments to investigate off-target effects, which have long time been overused. Performing similar analysis, but using "global experiments", such as, RNA-seq, to identify signatures specific to electrophile-target engagement, and comparing these to similar data from cells treated with hybrid inhibitors could be even more transformative; profile changes observed upon treatment with electrophile-reduced hybrid drug could obviously be included. Such signatures, if performed in sufficient cell lines, or in pertinent model organisms could further help triage hybrid drugs at earlier stages in the pipeline, winnowing pools of inhibitors to those showing the best on-target profiles.

\section{Prospects}

In sum, despite issues associated with the use of fusion proteins, oftentimes overexpressed or at least expressed above the wild-type background, T-REX offers insights that are complementary not only to electrophile-signaling discovery research but drug discovery in general. Furthermore, its applicability to a range of model organisms positions T-REX to be ideally useful as a bridge between basic laboratory science and more advanced studies leading into trials. It remains to be seen how predictive T-REX can 
generally be, but defining target-specific engagement signatures, potentially even as a function of time post engagement, could truly be a revolutionary weapon in drug discovery.

Author contributions: concept and writing: M.J.C.L., Y.A.; figures: A.K.; references: all authors. All authors agree to the final version of the manuscript. Funding: EPFL, SNSF project funding 184729, SNSF NCCR Chemical Biology.

\section{References}

[1] B. J. Druker, M. Talpaz, D. J. Resta, B. Peng, E. Buchdunger, J. M. Ford, N. B. Lydon, H. Kantarjian, R. Capdeville, S. Ohno-Jones, C. L. Sawyers, N. Engl. J. Med. 2001, 344, 1031-1037.

[2] D. Kazandjian, G. M. Blumenthal, W. Yuan, K. He, P. Keegan, R. Pazdur, Clin. Cancer Res. Off. J. Am. Assoc. Cancer Res. 2016, 22, 1307-1312.

[3] P. Valent, C. Akin, K. Hartmann, T. I. George, K. Sotlar, B. Peter, K. V. Gleixner, K. Blatt, W. R. Sperr, P. W. Manley, O. Hermine, H. C. Kluin-Nelemans, M. Arock, H.-P. Horny, A. Reiter, J. Gotlib, Ann. Oncol. 2017, 28, 2367-2376.

[4] Y. Sun, Y. Yang, Z. Wang, F. Jiang, Z. Chen, Z. Wang, Front. Pharmacol. 2020, 11, DOI 10.3389/fphar.2020.589146.

[5] D. Hoehn, J. E. Cortes, L. J. Medeiros, E. J. Jabbour, J. E. Hidalgo, R. Kanagal-Shamanna, C. E. Bueso-Ramos, Clin. Lymphoma Myeloma Leuk. 2016, 16, S86-S92.

[6] J. R. Poganik, Y. Aye, Front. Aging Neurosci. 2020, 12, DOI 10.3389/fnagi.2020.00001.

[7] A. P. Lawson, M. J. C. Long, R. T. Coffey, Y. Qian, E. Weerapana, F. E. Oualid, L. Hedstrom, Cancer Res. 2015, 75, 5130-5142.

[8] J. B. Fell, J. P. Fischer, B. R. Baer, J. F. Blake, K. Bouhana, D. M. Briere, K. D. Brown, L. E. Burgess, A. C. Burns, M. R. Burkard, H. Chiang, M. J. Chicarelli, A. W. Cook, J. J. Gaudino, J. Hallin, L. Hanson, D. P. Hartley, E. J. Hicken, G. P. Hingorani, R. J. Hinklin, M. J. Mejia, P. Olson, J. N. Otten, S. P. Rhodes, M. E. Rodriguez, P. Savechenkov, D. J. Smith, N. Sudhakar, F. X. Sullivan, T. P. Tang, G. P. Vigers, L. Wollenberg, J. G. Christensen, M. A. Marx, J. Med. Chem. 2020, 63, 6679-6693.

[9] J. Canon, K. Rex, A. Y. Saiki, C. Mohr, K. Cooke, D. Bagal, K. Gaida, T. Holt, C. G. Knutson, N. Koppada, B. A. Lanman, J. Werner, A. S. Rapaport, T. San Miguel, R. Ortiz, T. Osgood, J.-R. Sun, X. Zhu, J. D. McCarter, L. P. Volak, B. E. Houk, M. G. Fakih, B. H. O'Neil, T. J. Price, G. S. Falchook, J. Desai, J. Kuo, R. Govindan, D. S. Hong, W. Ouyang, H. Henary, T. Arvedson, V. J. Cee, J. R. Lipford, Nature 2019, 575, 217-223.

[10] Mirati Therapeutics Inc., A Phase 2 Trial of MRTX849 in Combination With Pembrolizumab in Patients With Advanced Non-Small Cell Lung Cancer With KRAS G12C Mutation, Clinicaltrials.Gov, 2021.

[11] Amgen, A Phase 1/2, Open-Label Study Evaluating the Safety, Tolerability, Pharmacokinetics, Pharmacodynamics, and Efficacy of AMG 510 Monotherapy in Subjects With Advanced Solid Tumors With KRAS p.G12C Mutation and AMG 510 Combination Therapy in Subjects With Advanced NSCLC With KRAS p.G12C Mutation (CodeBreak 100), Clinicaltrials.Gov, 2021.

[12] M. Visscher, M. R. Arkin, T. B. Dansen, Curr. Opin. Chem. Biol. 2016, 30, 61-67.

[13] M. J. C. Long, Y. Aye, Cell Chem. Biol. 2017, 24, 787-800.

[14] M. J. C. Long, L. Wang, Y. Aye, Antioxid. Redox Signal. 2019, 33, 1077-1091.

[15] P. Sarkar, V. Yarlagadda, C. Ghosh, J. Haldar, MedChemComm 2017, 8, 516-533.

[16] D. Chen, M. Frezza, S. Schmitt, J. Kanwar, Q. P. Dou, Curr. Cancer Drug Targets 2011, 11, 239253.

[17] M. L. Khan, A. K. Stewart, Future Oncol. Lond. Engl. 2011, 7, 607-612.

[18] H. Esterbauer, R. J. Schaur, H. Zollner, Free Radic. Biol. Med. 1991, 11, 81-128.

[19] H. Esterbauer, J. Gebicki, H. Puhl, G. Jürgens, Free Radic. Biol. Med. 1992, 13, 341-390.

[20] T. Ishikawa, H. Esterbauer, H. Sies, J. Biol. Chem. 1986, 261, 1576-1581. 
[21] W. G. Siems, H. Zollner, T. Grune, H. Esterbauer, J. Lipid Res. 1997, 38, 612-622.

[22] N. Zarkovic, Z. Ilic, M. Jurin, R. J. Schaur, H. Puhl, H. Esterbauer, Cell Biochem. Funct. 1993, 11, 279-286.

[23] L. I. Szweda, K. Uchida, L. Tsai, E. R. Stadtman, J. Biol. Chem. 1993, 268, 3342-3347.

[24] K. Uchida, E. R. Stadtman, Proc. Natl. Acad. Sci. U. S. A. 1992, 89, 4544-4548.

[25] K. Uchida, L. I. Szweda, H. Z. Chae, E. R. Stadtman, Proc. Natl. Acad. Sci. U. S. A. 1993, 90, 87428746.

[26] K. Uchida, K. Itakura, S. Kawakishi, H. Hiai, S. Toyokuni, E. R. Stadtman, Arch. Biochem. Biophys. 1995, 324, 241-248.

[27] M. J. Evans, B. F. Cravatt, Chem. Rev. 2006, 106, 3279-3301.

[28] M. J. Niphakis, B. F. Cravatt, Annu. Rev. Biochem. 2014, 83, 341-377.

[29] A. E. Speers, B. F. Cravatt, Curr. Protoc. Chem. Biol. 2009, 1, $29-41$.

[30] C. Wang, E. Weerapana, M. M. Blewett, B. F. Cravatt, Nat. Methods 2014, 11, 79-85.

[31] E. Weerapana, C. Wang, G. M. Simon, F. Richter, S. Khare, M. B. D. Dillon, D. A. Bachovchin, K. Mowen, D. Baker, B. F. Cravatt, Nature 2010, 468, 790-795.

[32] X. Liu, M. J. C. Long, Y. Aye, Trends Biochem. Sci. 2019, 44, 75-89.

[33] Y. Wang, M. M. Dix, G. Bianco, J. R. Remsberg, H.-Y. Lee, M. Kalocsay, S. P. Gygi, S. Forli, G. Vite, R. M. Lawrence, C. G. Parker, B. F. Cravatt, Nat. Chem. 2019, 11, 1113-1123.

[34] Y. Chen, Y. Liu, T. Lan, W. Qin, Y. Zhu, K. Qin, J. Gao, H. Wang, X. Hou, N. Chen, J. P. Friedmann Angeli, M. Conrad, C. Wang, J. Am. Chem. Soc. 2018, 140, 4712-4720.

[35] S. Parvez, M. J. C. Long, J. R. Poganik, Y. Aye, Chem. Rev. 2018, 118, 8798-8888.

[36] M. J. C. Long, C. Rogg, Y. Aye, Acc. Chem. Res. 2020, DOI 10.1021/acs.accounts.0c00537.

[37] M. J. C. Long, J. R. Poganik, Y. Aye, J. Am. Chem. Soc. 2016, 138, 3610-3622.

[38] S. L. Surya, M. J. C. Long, D. A. Urul, Y. Zhao, E. J. Mercer, I. M. Elsaid, T. Evans, Y. Aye, ACS Chem. Biol. 2018, 13, 1824-1831.

[39] M. J. C. Long, S. Parvez, Y. Zhao, S. L. Surya, Y. Wang, S. Zhang, Y. Aye, Nat. Chem. Biol. 2017, 13, 333-338.

[40] T. A. Yap, L. Yan, A. Patnaik, I. Fearen, D. Olmos, K. Papadopoulos, R. D. Baird, L. Delgado, A. Taylor, L. Lupinacci, R. Riisnaes, L. L. Pope, S. P. Heaton, G. Thomas, M. D. Garrett, D. M. Sullivan, J. S. de Bono, A. W. Tolcher, J. Clin. Oncol. 2011, 29, 4688-4695.

[41] Y. Xing, N. U. Lin, M. A. Maurer, H. Chen, A. Mahvash, A. Sahin, A. Akcakanat, Y. Li, V. Abramson, J. Litton, M. Chavez-MacGregor, V. Valero, S. A. Piha-Paul, D. Hong, K.-A. Do, E. Tarco, D. Riall, A. K. Eterovic, G. M. Wulf, L. C. Cantley, G. B. Mills, L. A. Doyle, E. Winer, G. N. Hortobagyi, A. M. Gonzalez-Angulo, F. Meric-Bernstam, Breast Cancer Res. 2019, 21, 78.

[42] X. Liu, M. J. C. Long, B. D. Hopkins, C. Luo, L. Wang, Y. Aye, ACS Cent. Sci. 2020, 6, 892-902.

[43] M. Rehan, M. A. Beg, S. Parveen, G. A. Damanhouri, G. F. Zaher, Plos One 2014, 9, e109705.

[44] K. Lin, J. Lin, W.-I. Wu, J. Ballard, B. B. Lee, S. L. Gloor, G. P. A. Vigers, T. H. Morales, L. S. Friedman, N. Skelton, B. J. Brandhuber, Sci. Signal. 2012, 5, ra37-ra37.

[45] M. J. C. Long, ChemBioChem n.d., n/a, DOI https://doi.org/10.1002/cbic.202000497. 